PROCEEDINGS OF THE

AMERICAN MATHEMATICAL SOCIETY

Volume 127, Number 4, April 1999, Pages 1195-1200

S 0002-9939(99)04950-3

\title{
STABLE CONSTANT MEAN CURVATURE SURFACES WITH CIRCULAR BOUNDARY
}

\author{
LUIS J. ALÍAS, RAFAEL LÓPEZ, AND BENNETT PALMER
}

(Communicated by Peter Li)

\begin{abstract}
In this paper we study stable constant mean curvature surfaces in the Euclidean space $\mathbf{R}^{3}$ with circular boundary. We show that in the case of genus zero, the only such surfaces are the spherical caps and the flat discs. We also extend this result to the case of surfaces in the other space forms, namely the sphere $\mathbf{S}^{3}$ and the hyperbolic space $\mathbf{H}^{3}$.
\end{abstract}

\section{INTRODUCTION}

The mathematical model of a soap bubble which has its boundary on a round hoop is a constant mean curvature surface with circular boundary. The surface forms in such a way as to minimize surface area while enclosing a fixed volume and spanning a fixed boundary. The surfaces we almost always observe are spherical caps and so it is natural to ask if these are the only solutions. Strictly speaking, the answer is no. Kapouleas [4] has shown that there exist high genus constant mean curvature (non-embedded) surfaces with circular boundaries. However, if we impose some additional restriction, such as that the surface is embedded or that it is a topological disc, the answer is unknown, although the problem has been treated by different authors in the last years. When the surface is embedded some partial results have been obtained in [2, 5, 8]; for the genus zero case, there are some answers in $[7,8]$, under certain hypotheses on the area of the surface.

Here we consider the question of the existence of stable constant mean curvature genus zero surfaces with boundary on a circle. We will assume, by rescaling if necessary, that the boundary is $\mathbf{S}^{1}:=\left\{\left(x_{1}, x_{2}, x_{3}\right) \in \mathbf{R}^{3}: x_{3}=0, x_{1}^{2}+x_{2}^{2}=1\right\}$.

Recall that a constant mean curvature surface $\Sigma$ is called stable if the second variation of area of the surface is non-negative for every smooth deformation of the surface which fixes the enclosed oriented three-volume and fixes the boundary. Let $J$ denote the corresponding Jacobi operator, that is, $J=\Delta+\|d \nu\|^{2}$, where $\Delta$ stands for the Laplacian operator of $\Sigma$. Then the stability of $\Sigma$ means that

$$
-\int_{\Sigma} f J[f] d A \geq 0
$$

Received by the editors July 24, 1997.

1991 Mathematics Subject Classification. Primary 53A10; Secondary 53C42.

The first author was partially supported by DGICYT Grant No. PB94-0750-C02-02 and Consejería de Educación y Cultura CARM Grant No. PB/5/FS/97, Programa Séneca (PRIDTYC).

The second author was partially supported by DGICYT Grant No PB94-0796.

The third author was supported by a DGICYT Grant No. SAB95-0494.

(C)1999 American Mathematical Society 
for all $f \in C_{0}^{\infty}(\Sigma)$ such that

$$
\int_{\Sigma} f d A=0
$$

We refer the reader to [1] for further details.

In this paper we will prove the following.

Theorem 1. Let $D$ denote the unit disc in the complex plane and let $X: D \longrightarrow \mathbf{R}^{3}$ be a conformal immersion with constant mean curvature $h$. Assume $X$ is stable and that $X(\partial D) \subset \mathbf{S}^{1}$. Then $X(D)$ is a spherical cap of radius $1 /|h|$. (The case $h=0$ corresponds to a flat disc.)

In relation to this, M. Koiso [6] has shown that for circular boundaries, spherical caps are the unique absolute minimizers for area enclosing a fixed volume.

\section{Proof of the theorem}

If the mean curvature is $h=0$, then, by the maximum principle, $X(D)$ is a flat disc, so we assume that $h \neq 0$.

Let $V$ be the vector field on $\mathbf{R}^{3}$ given by $V(x)=E_{3} \wedge x$ where $E_{3}$ denotes the vertical unit vector and $\wedge$ is the vector cross product. The field $V$ can be expressed as $V(x)=\alpha x$ with $\alpha \in s o(3)$. The one-parameter subgroup $e^{t \alpha}$ generates rotations about the vertical axis. If $X: D \longrightarrow \mathbf{R}^{3}$ is a constant mean curvature immersion, then $X_{t}:=e^{t \alpha} X$ gives a one-parameter family of such immersions and therefore the function

$$
\psi:=\left\langle\partial_{t}\left(X_{t}\right)_{t=0}, \nu\right\rangle=\left\langle E_{3} \wedge X, \nu\right\rangle,
$$

where $\nu$ is a unit normal to the immersion, defines a Jacobi field along $X$. This means that $\psi$ solves the equation

$$
0=J[\psi]:=\left(\Delta+\|d \nu\|^{2}\right) \psi
$$

If $X(\partial D) \subset \mathbf{S}^{1}$, then $X_{t}(\partial D) \subset \mathbf{S}^{1}$ for all $t$ and $\psi \equiv 0$ on $\partial D$. Further, by applying the divergence theorem to the Killing field $V$, it is easy to see that

$$
\int_{D} \psi d A=0
$$

which is the condition that the deformation of $X$ given by $X_{t}$ preserves the enclosed oriented 3-volume.

Our result will be esentially a consequence of the following statement.

Claim. The normal derivative of $\psi$ vanishes at least three times.

Let $z=x+i y=r e^{i \theta}$ be the usual coordinate in $D$. Then the first and second fundamental forms of the immersion can be expressed as

$$
d s^{2}=: e^{\mu}|d z|^{2}, \quad \mathrm{II}=: \operatorname{Re}\left\{\phi d z^{2}+h e^{\mu} d z d \bar{z}\right\},
$$

where $\phi d z^{2}$ is the Hopf differential of the immersion. It is well known that $\phi$ is holomorphic with respect to the local coordinate $z$. The unit tangent and unit normal to $\partial D$ with respect to $d s^{2}$ are given by

$$
t:=e^{-\mu / 2} \partial_{\theta} \quad \text { and } \quad n:=e^{-\mu / 2} \partial_{r} .
$$

Computing the normal derivative of $\psi$ on the boundary gives

$$
\partial_{n} \psi=\partial_{n}\left\langle E_{3} \wedge X, \nu\right\rangle=\left\langle E_{3} \wedge d X(n), \nu\right\rangle+\left\langle E_{3} \wedge X, d \nu(n)\right\rangle .
$$


Because the immersion is conformal, we have $\nu \wedge d X(n)=d X(t)$ and so

$$
\left\langle E_{3} \wedge d X(n), \nu\right\rangle=\left\langle E_{3}, d X(t)\right\rangle=0 .
$$

Also, by elementary geometry $E_{3} \wedge X=d X(t)$ on $\partial D$ and so

$$
\partial_{n} \psi=\langle d X(t), d \nu(n)\rangle=-\mathrm{II}(t, n)
$$

on $\partial D$.

On a neighborhood of any point on $\partial D$ we can choose a branch of the logarithm and make the holomorphic change of coordinate $w=\log z$. Since $w \equiv \log |z|+i \theta$ $(\bmod 2 \pi i)$, we have

$$
\partial_{w}=\frac{1}{2}\left(\partial_{r}-i \partial_{\theta}\right)
$$

globally on $\partial D$ since $\partial_{\theta}$ is independent of the branch of logarithm. Also note that $\partial_{w}=z \partial_{z}$ globally on $0<|z| \leq 1$. Therefore on $0<|z| \leq 1$ we have

$$
z^{2} \phi=z^{2} 2 \mathrm{II}\left(\partial_{z}, \partial_{z}\right)=2 \mathrm{II}\left(\partial_{w}, \partial_{w}\right)
$$

and on $|z|=1$ we have

$$
\operatorname{Im}\left(z^{2} \phi\right)=-\operatorname{II}\left(\partial_{r}, \partial_{\theta}\right) .
$$

Using (3) and (4) we therefore obtain

$$
\operatorname{Im}\left(z^{2} \phi\right)=e^{\mu} \partial_{n} \psi, \quad \text { on } \partial D .
$$

Let us see that there are at least three distinct points on $\partial D$ where $\operatorname{Im}\left(z^{2} \phi\right)=0$. In order to prove this we can assume that there are at most two distinct points $a_{1}, a_{2}$ on the boundary with $\phi\left(a_{j}\right)=0$. We will assume that there are exactly two points and we include the other cases by allowing zeros of zero order. Since $\phi$ has no singularities on $\partial D$, we can extend $\phi$ analytically to a slightly larger disc of radius $R>1$. Let $\varepsilon>0$ be a sufficiently small number so that $\phi^{-1}(0) \cap \bar{D}_{\varepsilon}\left(a_{j}\right)=\left\{a_{j}\right\}$, where $D_{\varepsilon}\left(a_{j}\right)=\left\{z:\left|z-a_{j}\right|<\varepsilon\right\}$.

Let $D_{\varepsilon}^{*}=D \backslash\left(D_{\varepsilon}\left(a_{1}\right) \cup D_{\varepsilon}\left(a_{2}\right)\right)$ and let $C_{\varepsilon}=\partial D_{\varepsilon}^{*}$ oriented positively (i.e. so that $D_{\varepsilon}^{*}$ always lies to the left). Since there are no zeros of $z^{2} \phi$ on $C_{\varepsilon}$, we have

$$
\frac{1}{i} \oint_{C_{\varepsilon}}\left(\frac{2}{z}+\frac{\phi^{\prime}}{\phi}\right) d z=4 \pi+2 \pi M(\varepsilon),
$$

where $M(\varepsilon)$ denotes the number of zeros of $\phi$ inside $C_{\varepsilon}$ with multiplicity. Note that the right hand side of (5) is the total variation of $\arg z^{2} \phi$ along the curve $C_{\varepsilon}$.

A standard residue calculation shows that

$$
\lim _{\varepsilon \downarrow 0} \frac{1}{i} \int_{C_{\varepsilon} \cap \partial D_{\varepsilon}\left(a_{j}\right)} \frac{\phi^{\prime}}{\phi} d z=-m_{j} \pi
$$

where $m_{j}$ denotes the order of the zero of $\phi$ at $a_{j}$. Therefore

$$
\frac{1}{i} \lim _{\varepsilon \downarrow 0} \int_{C_{\varepsilon} \backslash \partial\left(D_{\varepsilon}\left(a_{1}\right) \cup D_{\varepsilon}\left(a_{2}\right)\right)}\left(\frac{2}{z}+\frac{\phi^{\prime}}{\phi}\right) d z=4 \pi+2 \pi M+\pi\left(m_{1}+m_{2}\right)
$$

where $M$ denotes the number of umbilics, with multiplicities, in the interior of $D$.

If $m_{1}=m_{2}=0$, then the image of $\partial D$ under the map $z^{2} \phi$ has winding number at least 2 with respect to the origin. Hence $\operatorname{Im}\left(z^{2} \phi\right)$ vanishes in at least four distinct points on $\partial D$. If $m_{1}+m_{2}>0$ holds, then by (6) the total variation in $\arg z^{2} \phi$ over the two arcs determined by the $a_{j}$ 's is at least $5 \pi$. It is then easy to see that there must be at least three points on $\partial D$ where $\operatorname{Im}\left(z^{2} \phi\right)$ vanishes. This finishes the proof of the claim. 
Our objective now is to show that $\psi$ vanishes identically on $D$. In fact, if this is not the case, then by (2) $\psi$ changes sign. Let $\left\{\lambda_{j}\right\}_{j=1,2,3, \ldots}$ denote the Dirichlet eigenvalues for $J$ in $D$ listed in increasing order. It follows that $\psi$ does not correspond to $\lambda_{1}$ since an eigenfunction for $\lambda_{1}$ cannot change sign. On the other hand $\psi$ cannot belong to $\lambda_{2}$ either. To see this, recall [3] that an eigenfunction $f_{2}$ belonging to $\lambda_{2}$ can partition $D$ into at most two nodal domains whose common boundary can intersect $\partial D$ in either two or zero points. By the Hopf maximum principle, $f_{2}$ has non-vanishing normal derivative at all other points on $\partial D$ and we have claimed above that the normal derivative of $\psi$ vanishes at least three times. Thus $0>\lambda_{j}, j=1,2$. It is then possible to define a function $f$ as a linear combination of the first two eigenfunctions such that (1) holds but

$$
-\int f J[f] d A<0
$$

This means that the immersion is unstable.

Therefore, $\psi \equiv 0$ on $D$, which means that the vector field $V$ is tangent to the surface at every point. Its integral curves foliate the surface by circles which project to concentric circles in the plane $x_{3}=0$. It follows that $X$ defines a surface of revolution. Since the surface is a topological disc and has constant non-zero mean curvature, it is a spherical cap, which yields the result.

\section{THE CASE OF OTHER SPACE FORMS}

In this section we will consider the same problem for surfaces in the remaining space forms, namely the sphere and the hyperbolic space. The main ideas of our proof also work here, so that we will briefly sketch the proof.

Let $\mathbf{S}^{3}=\left\{x \in \mathbf{R}^{4}:|x|=1\right\} \subset \mathbf{R}^{4}$ be the three-dimensional unit sphere and let $X: D \longrightarrow \mathbf{S}^{3} \subset \mathbf{R}^{4}$ be a conformal immersion with constant mean curvature $h$ and circular boundary. This means that $X(\partial D) \subset \mathbf{S}^{1}(r)$, where we may assume, by rotating $\mathbf{S}^{3}$ in $\mathbf{R}^{4}$ if necessary, that the boundary is

$$
\mathbf{S}^{1}(r)=\left\{\left(x_{1}, x_{2}, x_{3}, x_{4}\right) \in \mathbf{S}^{3}: x_{3}=0, x_{4}^{2}=1-r^{2}, x_{1}^{2}+x_{2}^{2}=r^{2}\right\}, \quad 0<r \leq 1 .
$$

In this case $V$ is the vector field on $\mathbf{S}^{3}$ given by $V(x)=E_{3} \wedge E_{4} \wedge x$, where $\wedge$ is the vector cross product in $\mathbf{R}^{4}$ determined by

$$
\left\langle v_{1} \wedge v_{2} \wedge v_{3}, v\right\rangle=\operatorname{det}\left(v_{1}, v_{2}, v_{3}, v\right),
$$

det being the determinant in the canonical basis. The field $V$ corresponds now to the one-parameter subgroup $e^{t \alpha}$ of rotations of $\mathbf{R}^{4}$ around the plane passing through the origin and generated by $E_{3}$ and $E_{4}$. If $X: D \longrightarrow \mathbf{S}^{3} \subset \mathbf{R}^{4}$ is a constant mean curvature immersion with $X(\partial D) \subset \mathbf{S}^{1}(r)$, then $X_{t}=e^{t \alpha}$ gives a one-parameter family of such immersions and the function

$$
\psi:=\left\langle\partial_{t}\left(X_{t}\right)_{t=0}, \nu\right\rangle=\left\langle E_{3} \wedge E_{4} \wedge X, \nu\right\rangle,
$$

where $\nu$ is a unit normal to $X$ in $\mathbf{S}^{3}$, defines a Jacobi field along $X$. That is, $\psi$ solves the equation

$$
J[\psi]:=\left(\Delta+\|d \nu\|^{2}+2\right) \psi=0,
$$

and $\psi \equiv 0$ on $\partial D$. The divergence theorem applied to the Killing field $V$ also gives

$$
\int_{D} \psi d A=0
$$


which is the condition that the deformation of $X$ given by $X_{t}$ preserves the enclosed oriented 3-volume.

As for the three-dimensional unit hyperbolic space $\mathbf{H}^{3}$, we will consider the Minkowski model given by $\mathbf{H}^{3}=\left\{x \in \mathbf{L}^{4}:\langle x, x\rangle=-1\right\} \subset \mathbf{L}^{4}$, where $\langle$,$\rangle stands$ now for the Lorentzian inner product in $\mathbf{L}^{4}$ with signature $(+++-)$. In this case, up to a rigid motion of $\mathbf{H}^{3}$ in $\mathbf{L}^{4}$, we may assume that the circular boundary $\mathbf{S}^{1}(r)$ is

$$
\mathbf{S}^{1}(r)=\left\{\left(x_{1}, x_{2}, x_{3}, x_{4}\right) \in \mathbf{H}^{3}: x_{3}=0, x_{4}^{2}=1+r^{2}, x_{1}^{2}+x_{2}^{2}=r^{2}\right\}, \quad r>0 .
$$

The vector field $V$ is now the Killing vector field on $\mathbf{H}^{3}$ given by $V(x)=E_{3} \wedge E_{4} \wedge x$, where $\wedge$ stands now for the Lorentz vector cross product in $\mathbf{L}^{4}$, which is determined by

$$
\left\langle v_{1} \wedge v_{2} \wedge v_{3}, v\right\rangle=\operatorname{det}\left(v_{1}, v_{2}, v_{3}, v\right),
$$

but using now the Lorentzian inner product. Let $X: D \longrightarrow \mathbf{H}^{3} \subset \mathbf{L}^{4}$ be a constant mean curvature immersion with $X(\partial D) \subset \mathbf{S}^{1}(r)$ and let $\nu$ be a unit normal to $X$ in $\mathbf{H}^{3}$. The same reasoning as above implies now that the function

$$
\psi:=\left\langle\partial_{t}\left(X_{t}\right)_{t=0}, \nu\right\rangle=\left\langle E_{3} \wedge E_{4} \wedge X, \nu\right\rangle
$$

is a Jacobi field along $X$. That is, $\psi$ solves the equation

$$
J[\psi]:=\left(\Delta+\|d \nu\|^{2}-2\right) \psi=0,
$$

and $\psi \equiv 0$ on $\partial D$, and it also satisfies

$$
\int_{D} \psi d A=0
$$

As in the case of Euclidean space, the idea is to see that $\psi$ vanishes identically on $D$. Following the proof above, it suffices to show now that the normal derivative of $\psi$ vanishes again at least three times. Computing the normal derivative of $\psi$ gives

$$
\partial_{n} \psi=\partial_{n}\left\langle E_{3} \wedge E_{4} \wedge X, \nu\right\rangle=\left\langle E_{3} \wedge E_{4} \wedge d X(n), \nu\right\rangle+\left\langle E_{3} \wedge E_{4} \wedge X, d \nu(n)\right\rangle .
$$

Since the immersion is conformal, now we have $\nu \wedge d X(n) \wedge X=d X(t)$, so that on the boundary we get that $E_{3} \wedge E_{4} \wedge d X(n)$ and $E_{3} \wedge E_{4} \wedge X$ are both collinear to $d X(t)$. Therefore,

$$
\partial_{n} \psi= \pm r \operatorname{II}(t, n) .
$$

The rest of the proof works as in the Euclidean case.

Corollary 2. Let $D$ denote the unit disc in the complex plane and let $X$ be a conformal immersion of $D$ into $\mathbf{S}^{3}$ or $\mathbf{H}^{3}$ with constant mean curvature. Assume $X$ is stable and that $X(\partial D) \subset \mathbf{S}^{1}(r)$. Then $X(D)$ is a geodesic sphere of a totally umbilic surface.

\section{ACKNOWLEDGEMENTS}

The second author would like to thank Professor Sebastián Montiel for useful discussions on the subject. This paper was written while the third author was visiting the Departamento de Matematicas of the Universidad de Murcia, Spain. He would like to thank that institution for its hospitality. 


\section{REFERENCES}

[1] J.L Barbosa And M. Do Carmo, Stability of hypersurfaces with constant mean curvature, Math. Z., 185 (1984), 339-353. MR 85k:58021c

[2] F. Brito, R. sa Earp, W.H. Meeks III and H. Rosenberg, Structure theorems for constant mean curvature surfaces bounded by a planar curve, Indiana Univ. Math. J., 40 (1991), 333343. MR 93e:53009

[3] R. Courant and D. Hilbert, Methods of Mathematical Physics, Volume I, John Wiley and Sons, New York, 1989. MR 16:426a (1953 edition)

[4] N. Kapouleas, Compact constant mean curvature surfaces in Euclidean three-space, J. Diff. Geom., 33 (1991), 683-715. MR 93a:53007b

[5] M. KoIso, Symmetry of hypersurfaces of constant mean curvature with symmetric boundary, Math. Z., 191 (1986), 567-574. MR 87f:53006

[6] M. Koiso, A generalization of Steiner symmetrization for immersed surfaces and its applications, Manuscripta Math., 87 (1995), 311-325. MR 96f:53013

[7] R. López And S. Montiel, Constant mean curvature discs with bounded area, Proc. Amer. Math. Soc., 123 (1995), 1555-1558. MR 95f:53016

[8] R. López and S. Montiel, Constant mean curvature surfaces with planar boundary, Duke Math. J., 85 (1996), 583-604. MR 97m:53015

Departamento de Matemáticas, Universidad de Murcia, 30100 Espinardo, Murcia, SPAIN

E-mail address: ljalias@fcu.um.es

Departamento de Geometría y Topología, Universidad de Granada, 18071 Granada, SPAIN

E-mail address: rcamino@ugr.es

Department of Mathematical Sciences, University of Durham, Durham DH1 3LE, ENGLAND

E-mail address: bennett.palmer@durham.ac.uk 